\title{
Dinámica trófica del jurel (Trachurus symmetricus murphyi) en el norte de Chile
}

\author{
Marianela Medina ${ }^{1}$ y Hugo Arancibia ${ }^{2}$ \\ ${ }^{1}$ Departamento de Ciencias del Mar, Universidad Arturo Prat. Casilla 121, Iquique, Chile \\ E-mail: mmedina@unap.cl \\ ${ }^{2}$ Departamento de Oceanografía, Universidad de Concepción. Casilla 160-C, Concepción, Chile \\ E-mail: harancib@udec.cl
}

Recibido: 24 septiembre 2001; versión corregida: 25 abril 2002; aceptado: 6 mayo 2002

\begin{abstract}
RESUMEN. Se determinó la dinámica trófica del jurel Trachurus symmetricus murphyi capturado frente a las costas de la zona norte de Chile, desde la primavera de 1993 hasta el invierno de 1994. Se consideró la importancia relativa de las presas, la identificación de unidades ontogenéticas tróficas y la selectividad de presas por tamaño. Las muestras se obtuvieron de las líneas de descarga de las plantas pesqueras en los puertos de Arica, Iquique, Tocopilla y Mejillones. En el laboratorio se identificaron, separaron, pesaron y contaron las presas de acuerdo a métodos estándar. En términos de importancia relativa, los eufáusidos fueron las principales presas en todas las estaciones del año analizadas, destacando también el pez mesopelágico Vinciguerria sp. Se identificaron tres unidades ontogenéticas tróficas, con límites en 21 y $35 \mathrm{~cm}$ de longitud horquilla. El análisis de selectividad de presas por tamaño indicó que la presa de mayor tamaño en el contenido estomacal fue Engraulis ringens. Además, ésta fue la presa más seleccionada junto a los quetognatos. La distribución de frecuencias de tamaños de E. ringens en los estómagos de jurel se sobrepone sólo parcialmente con la distribución de frecuencias de tamaños de E. ringens en las capturas comerciales, siendo en el primer caso de menor tamaño que en el segundo.
\end{abstract}

Palabras claves: jurel, Trachurus symmetricus murphyi, alimentación, norte de Chile.

\section{Trophic dynamic of jack mackerel (Trachurus symmetricus murphyi) in northern Chile}

\begin{abstract}
The trophic dynamic of jack mackerel Trachurus symmetricus murphyi, caught off the coast of northern Chile, between the spring 1993 and winter 1994, is described. Relative importance of prey taxa, identification of ontogenetic trophic units and prey selectivity by size was evaluated. The main prey in terms of relative importance were euphausids, for all seasons analyzed. Another important prey was mesopelagic fish Vinciguerria sp. Three ontogenetic trophic units were identified, within the size range of 21 to $35 \mathrm{~cm}$ fork length. The selectivity analysis of preys by size indicated that the large size prey in the stomach content was Engraulis ringens. Besides, this prey was the more selected together with Chaetognatha. The size frequency distribution of $E$. ringens in the stomach of jack mackerel overlapped partially with the sizes frequency distribution of $E$. ringens obtained from the commercial captures, being in the first case of smaller size that in the second.
\end{abstract}

Key words: jack mackerel, Trachurus symmetricus murphyi, feeding, northern Chile.

\section{INTRODUCCIÓN}

Los estudios de alimentación han sido reconocidos como importante fuente de información para análisis complementarios de los recursos pesqueros, relacionándolos con la distribución de tales recursos en el tiempo o espacio, migraciones, consumo y mortalidad por predación (Gislason y Sparre, 1987; Caddy y Sharp, 1988; Arancibia, 1991; Prenski y Angelescu, 1993). Además, la tendencia en el manejo pesquero requiere entender el rol de las especies predadoras dentro del ecosistema y, más 
específicamente, entender el efecto que la predación tiene sobre poblaciones explotadas, requiriéndose investigaciones pesqueras con integración ecotrófica (Caddy y Sharp, 1988; Daan y Sissenwine, 1991; Prenski y Angelescu, 1993).

En este contexto, en la actualidad existe un consenso general surgido entre los científicos y administradores pesqueros que las aproximaciones tradicionales en manejo pesquero especie-específica deben ser reemplazadas por un manejo ecosistémico, que considera explícitamente las interacciones ecológicas, especialmente aquellas de naturaleza trófica, como las relaciones interespecíficas e influencias ambientales (Caddy y Sharp, 1988; Walters et al., 1997; Bax, 1998).

Trachurus symmetricus murphyi es un recurso pesquero pelágico con distribución en el Océano Pacífico Sur, desde Ecuador ( $1^{\circ} 30^{\prime}$ S ) hasta el sur de Chile $\left(55^{\circ} \mathrm{S}\right)$, alcanzando hacia el oeste las costas de Nueva Zelandia, Tasmania y Australia (Serra, 1991; Grechina, 1992), se encuentra principalmente entre los $30^{\circ}$ y $50^{\circ} \mathrm{S}$ constituyendo lo que se conoce como el "cinturón del jurel". T. s. murphyi ha sido descrito como un predador facultativo en el ecosistema pelágico de Perú (Konchina, 1980, 1983), debido a su naturaleza migratoria, accede a ambientes neríticos y oceánicos epipelágicos para alimentarse, lo que ha sido confirmado por Medina y Arancibia (1992) para el norte de Chile. Serra (1991) describe para la subpoblación chilena de $T$. s. murphyi la existencia de una migración estacional hacia el océano abierto para su reproducción y desde allí al área costera para alimentarse, lo que configura un patrón que determina la disponibilidad estacional del recurso en las pesquerías costeras y oceánicas, y constituye un factor importante para la evaluación de su stock.

La composición alimentaria del jurel en el norte de Chile indica que sus principales presas son eufáusidos y peces mesopelágicos: mictófidos y gonostomátidos (Medina y Arancibia, 1992). Para esta especie, los cambios ontogénicos en su dieta no han sido muy claros, pero sí para otras especies de peces marinos y de agua dulce (Ross, 1978; Livingston, 1982; Arancibia, 1989), donde los cambios en los hábitos alimenticios han sido asociados con cambios ontogénicos en la morfología del hocico, dimensiones del cuerpo, primera madurez sexual y alteración progresiva de atributos en las conductas individuales.

Considerando la importancia que está tomando la modelación ecotrófica de los ecosistemas marinos que permite comprender el rol de cada especie en el ecosistema, se ha planteado como objetivos determinar la importancia relativa de las presas, identificar las unidades ontogenéticas tróficas y analizar la selectividad de presas por tamaño en el jurel por ser un componente importante dentro del ecosistema pelágico del norte de Chile.

\section{MATERIALES Y MÉTODOS}

El área de estudio comprendió desde Arica (18²1' S) hasta el sur de Antofagasta (233' S), donde opera habitualmente la flota industrial de cerco de la zona norte. Los muestreos de jurel se realizaron en las líneas de descarga en Arica, Iquique, Tocopilla y Mejillones, desde primavera de 1993 hasta invierno de 1994. El tamaño mínimo de muestra se calculó de acuerdo con Pennington et al. (1982). Los ejemplares se midieron, sexaron y pesaron, y los estómagos extraídos se conservaron en formalina al $10 \%$ para su posterior análisis. En el laboratorio se identificaron, separaron, contaron y pesaron las presas (peso húmedo con 0,0001 g de precisión). En el caso de los peces como presas, se registró la longitud total.

La importancia relativa de cada taxon presa en la dieta se determinó con el Indice de Importancia Relativa (IIR) (Pinkas et al., 1971 fide Hyslop, 1980), a pesar que ha sido criticado debido a su supuestamente cuestionable importancia biológica. No obstante, se escogió porque es una medida práctica de la importancia trófica que incorpora el número relativo de organismos, relevante en la teoría de forraje y el porcentaje del volumen o peso es un probable reflejo del contenido calórico del alimento (Wallace, 1981 fide Schmitter-Soto y Castro-Aguirre, 1996) facilitando, además, los estudios comparativos. En este estudio se ha utilizando la expresión modificada por Arancibia et al. (1995) a la forma logarítmica, para disminuir las excesivas diferencias numéricas entre valores, cuya expresión es:

$$
\mathrm{IIR}=\ln ((\mathrm{N}+\mathrm{W}) \times \mathrm{F}+1)
$$

donde $\mathrm{N}$ es el porcentaje en número; W es el porcentaje en peso, y $\mathrm{F}$ es el porcentaje de frecuencia de ocurrencia de cada taxon.

En la determinación previa de las unidades ontogenéticas tróficas, esto es, clases de tallas con similar base trófica (sensu Livingston, 1982), se uti- 
lizó el Indice de Similitud de Bray-Curtis (Bloom, 1981) y el criterio de unión de grupos de la media aritmética se efectuó por pares no ponderados (Sneath y Sokal, 1973). Inicialmente, se consideraron clases de tallas cada $5 \mathrm{~cm}$ de longitud horquilla. La variable utilizada fue el peso de las presas (en porcentaje) y se eliminaron los taxa de presas cuya contribución en peso a la muestra total fue menor de 1\% considerándolas incidentales (Ross, 1978). El Indice de Similitud (IS) tiene la siguiente forma:

$$
I S=\frac{2 \sum_{i=1}^{m} m n\left(X_{i j}, X_{k j}\right)}{\sum_{i=1}^{m}\left(X_{i j}+X_{k j}\right)}
$$

donde: mín es el peso menor entre $\mathrm{X}_{\mathrm{ij}}$ y $\mathrm{X}_{\mathrm{kj}}, \mathrm{X}_{\mathrm{ij}}$ es la ocurrencia (porcentaje en peso) del j-ésimo taxon de presa en el i-ésimo grupo de tamaño del predador; $\mathrm{X}_{\mathrm{kj}}$ es la ocurrencia (porcentaje en peso) del mismo taxon de presa en el k-ésimo grupo de tamaño del predador.

La selectividad de presas por tamaño fue determinada usando la metodología de Ursin (1973), donde el tamaño relativo de cada presa en el estómago de cada predador es calculado como la razón entre el peso del predador y el peso de la presa, y cada razón es designada como un valor ISU. Las preferencias se reflejan como una distribución de frecuencias derivada del logaritmo natural de sus pesos cuya forma es:

$$
\mathrm{ISU}=\operatorname{Ln}\left(\frac{\mathrm{w}_{\mathrm{i}}}{\mathrm{w}_{\mathrm{j}}}\right)
$$

donde $\mathrm{w}_{\mathrm{i}}$ es el peso del predador $\mathrm{i}(\mathrm{g})$; $\mathrm{w}_{\mathrm{j}}$ es el peso del taxon de presa $\mathrm{j}(\mathrm{g})$ en el estómago del predador i. De acuerdo con Clark (1985), $\mathrm{w}_{\mathrm{j}}$ corresponde al peso de cada taxon en el contenido estomacal de cada pez y no al peso de cada presa individual por pez, ya que considera que la presencia de sólo una o unas pocas presas planctónicas en el estómago del predador es el resultado de un simple encuentro entre el predador y la presa. Los peces predan sobre una mancha de pequeñas presas planctónicas (bulk prey) y no sobre pequeños plancteres en forma individual (Arancibia, 1989).

Los valores ISU fueron agrupados en intervalos de 1,0 y graficados para ver si la distribución de frecuencias es estrictamente una normal. Se considera que el promedio de los valores del índice de selectividad (ISU) representa el tamaño promedio entre el predador y sus presas, y que la desviación estándar es una medida relativa de la selectividad, siendo inversamente proporcional a ésta.

\section{RESULTADOS}

El número mínimo de muestras fue calculado por trimestre en 1.350 estómagos de jurel y se analizó un total de 7.444 estómagos, donde sólo el $24 \%$ (1.784 estómagos) tenían contenido. El análisis estacional de la importancia relativa de las presas de jurel indica que, en primavera de 1993, los eufáusidos son la presa más importante, aunque también destacaron copépodos, larvas de crustáceos, $E$. ringens y Vinciguerria sp. En verano de 1994, los eufáusidos y Vinciguerria sp. fueron las presas más importantes. Finalmente, en otoño e invierno nuevamente los eufáusidos fueron la presa más importante, seguida de E. ringens (Tablas 1 a 4).

La composición trófica en el jurel por clase de talla y estación del año se presentan en la figura 1. En primavera de 1993, la composición del alimento en todas las clases de tallas superiores a $20 \mathrm{~cm}$ de longitud horquilla (LH) fue dominada por eufáusidos, E. ringens y Vinciguerria sp.; este último taxon fue también importante en los individuos menores a $21 \mathrm{~cm}$ de LH, aunque con fuerte presencia de Brachyura. Se destacó también la presencia de copépodos en individuos menores a $36 \mathrm{~cm} \mathrm{LH}$. En verano de 1994 predominó Vinciguerria sp. como presa en las tres clases de talla muestreadas, aunque también estuvieron representados fuertemente los eufáusidos. En los individuos mayores de $30 \mathrm{~cm} \mathrm{LH}$ se destacó, además, la presencia de E. ringens. En otoño predominaron los eufáusidos en las cuatro clases de talla muestreadas, con una fuerte presencia de E. ringens en los ejemplares mayores a 20 cm LH. Finalmente, en invierno, la composición del alimento en las tres clases de talla muestreadas estuvo dominada por eufáusidos, con presencia de $E$. ringens en individuos mayores a $25 \mathrm{~cm} \mathrm{LH}$. Vinciguerria sp. presentó una alta importancia relativa en los individuos menores a $26 \mathrm{~cm} \mathrm{LH}$

Se reconocieron tres unidades ontogenéticas tróficas en jurel en primavera de 1993, una constituida por individuos menores a $21 \mathrm{~cm}$ de LH (Grupo 1), otra entre 21 y $35 \mathrm{~cm}$ (Grupos 2, 3 y 4), y la última conformada por peces mayores a $35 \mathrm{~cm}$ (Grupos 5 y 6) (Fig. 2a). La similitud trófica promedio fue de 0,56. La segregación de los individuos me- 


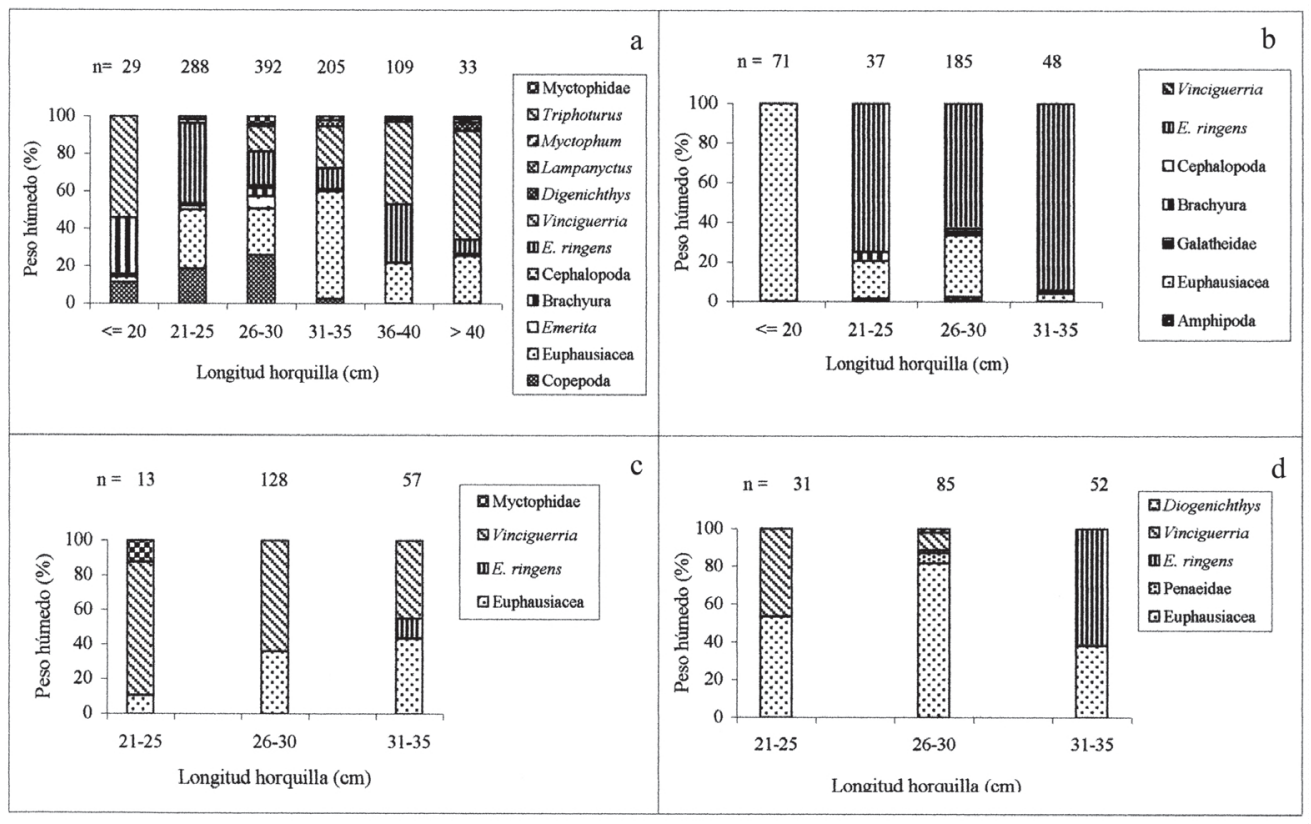

Figura 1. Composición de la dieta (en porcentaje de peso húmedo) por grupo de talla en jurel del norte de Chile por estación. a) primavera 1993, b) otoño 1994, c) verano 1994 y d) invierno 1994.

Figure 1. Seasonal diet composition (as percentage wet weight) by length group in jack mackerel in northern Chile. a) spring 1993, b) autumm 1994, c) summer 1994 and d) winter 1994.

Tabla 1. Porcentaje en número $(\% \mathrm{~N})$, porcentaje en peso $(\% \mathrm{P})$, porcentaje de frecuencia de ocurrencia $(\% \mathrm{~F})$ e índice de importancia relativa por ítemes presa de jurel en primavera de 1993.

Table 1. Percentage in numbers $(\% \mathrm{~N})$, in weight $(\% \mathrm{P})$, of occurrence frequency $(\% \mathrm{~F})$ and index of relative importance, by prey taxon of jack mackerel for the spring 1993.

\begin{tabular}{|lrrrr|}
\hline PRESAS & \%N & $\mathbf{\% P}$ & $\mathbf{\%}$ & IIR \\
\hline CRUSTACEA & & & & \\
Copepoda & 62,98 & 7,86 & 8,71 & 6,43 \\
Ostracoda & 0,66 & 0,20 & 7,83 & 2,04 \\
Amphipoda & 1,06 & 0,13 & 5,03 & 1,94 \\
Estomatopoda & 0,65 & 0,07 & 2,18 & 0,94 \\
Euphausiacea & 20,17 & 31,38 & 24,62 & 7,15 \\
Penaeidae & 0,24 & 0,06 & 1,04 & 0,27 \\
Emerita sp. & 5,39 & 1,67 & 7,57 & 4,00 \\
Blepharipoda & 0,07 & 0,01 & 0,26 & 0,02 \\
Lepidopa & 0,23 & 0,03 & 1,35 & 0,29 \\
Galatheidae & 0,04 & 0,03 & 0,36 & 0,02 \\
Paguridae & 0,10 & 0,01 & 1,40 & 0,14 \\
Porcellanidae & 0,17 & 0,03 & 1,35 & 0,24 \\
Anomura & 0,06 & 0,01 & 1,04 & 0,07 \\
Brachyura & 4,07 & 1,13 & 18,09 & 4,56 \\
Crustáceos indetermin. & 0,00 & 0,01 & 0,05 & 0,00 \\
& & & & \\
& & & & \\
\hline
\end{tabular}

\begin{tabular}{|lrrrr|}
\hline PRESAS & $\mathbf{\%}$ & $\mathbf{\%} \mathbf{P}$ & $\mathbf{\%} \mathbf{F}$ & IIR \\
\hline MOLLUSCA & & & & \\
Pteropoda & 0,84 & 0,22 & 5,96 & 1,99 \\
Cephalopoda Teuthoidea & 0,01 & 0,57 & 0,41 & 0,22 \\
CHAETOGNATHA & 1,56 & 0,33 & 2,07 & 1,59 \\
UROCHORDATA & & & & \\
Salpidae & 0,00 & 0,17 & 0,10 & 0,02 \\
PISCES & & & & \\
E. ringens & 0,04 & 22,59 & 2,49 & 4,05 \\
Vinciguerria sp. & 1,33 & 26,87 & 2,54 & 4,29 \\
Astronesthidae & 0,00 & 0,10 & 0,05 & 0,01 \\
Notosudidae & 0,00 & 0,19 & 0,05 & 0,01 \\
Diogenichthys sp. & 0,12 & 1,30 & 1,81 & 1,27 \\
Lampanyctus sp. & 0,00 & 0,65 & 0,16 & 0,10 \\
Myctophum sp. & 0,00 & 0,60 & 0,21 & 0,12 \\
Symbolophorus sp. & 0,00 & 0,29 & 0,10 & 0,03 \\
Triphoturus sp. & 0,01 & 0,42 & 0,47 & 0,18 \\
Myctophidae indet. & 0,03 & 1,81 & 1,09 & 1,10 \\
N. crockeri & 0,00 & 0,04 & 0,05 & 0,00 \\
Teleósteos indetermin. & 0,16 & 1,23 & 1,56 & 1,15 \\
& & & & \\
\hline
\end{tabular}



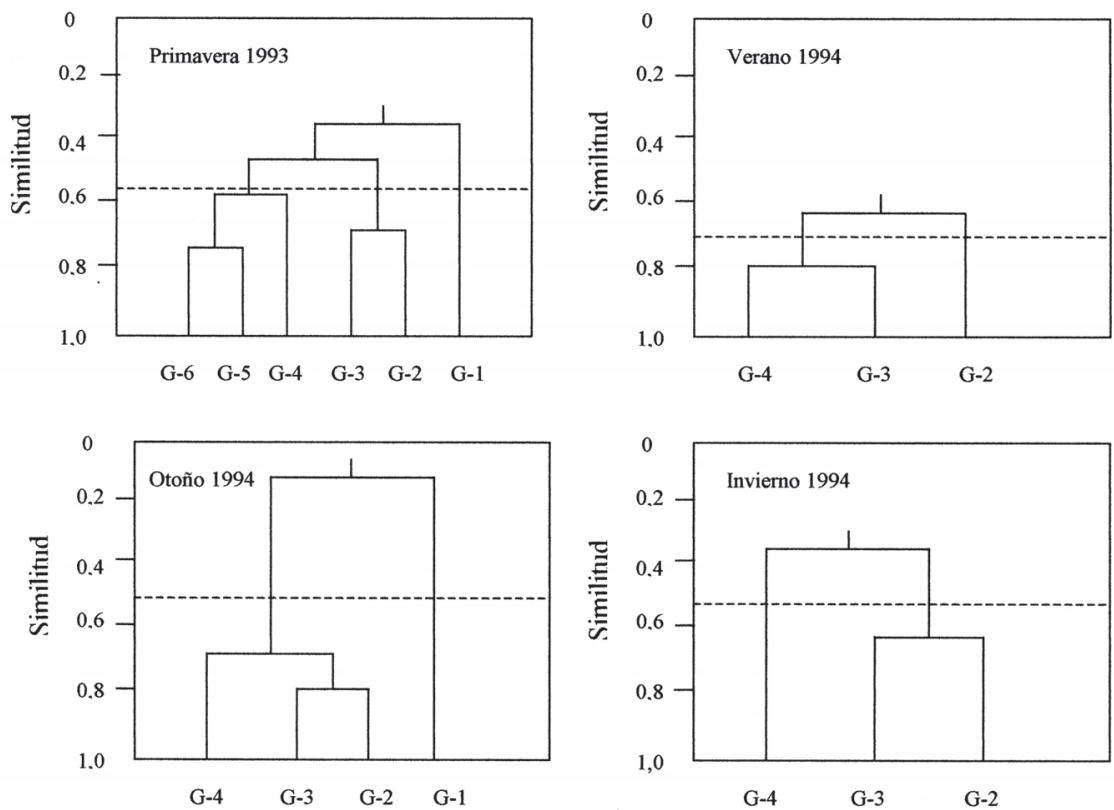

Figura 2. Dendrogramas de similitud trófica estacional de jurel en la zona norte de Chile en primavera de 1993; verano, otoño e invierno de 1994. Cada grupo de talla es de 5 cm de LH. G-1: - 20; G-2: 21-25; G-3: 26-30; G4: 31-35; G-5: 36-40; G- 6: • 40. La línea horizontal de trazos indica el promedio del índice de similitud.

Figure 2. Seasonal trophic similarity dendrograms in jack mackerel in northern Chile, for spring 1993; summer, autumn and winter 1994. Each size group has an amplitude of $5 \mathrm{~cm}$ FL. G-1: - 20; G-2: 21-25; G-3: 26-30; G-4: 31-35; G-5: 36-40; G-6: • 40. The broken line shows the average value of the similarity index.

Tabla 2. Porcentaje en número $(\% \mathrm{~N})$, porcentaje en peso $(\%)$, porcentaje de frecuencia de ocurrencia (\%) e índice de importancia relativa por ítemes presa de jurel en verano de 1994.

Table 2. Percentage in numbers $(\% \mathrm{~N})$, in weight $(\% \mathrm{P})$, of occurrence frequency $(\% \mathrm{~F})$ and index of relative importance, by prey taxon of jack mackerel for the summer 1994.

\begin{tabular}{|lrrrr|}
\hline PRESAS & $\mathbf{\% N}$ & $\mathbf{\%}$ & $\mathbf{\%}$ & IIR \\
\hline CRUSTACEA & & & & \\
Ostracoda & 0,25 & 0,02 & 1,33 & 0,31 \\
Euphausiacea & 83,69 & 33,67 & 53,98 & 8,75 \\
Emerita sp. & 0,03 & 0,00 & 0,44 & 0,01 \\
Galatheidae & 0,67 & 0,03 & 0,88 & 0,48 \\
Brachyura & 8,07 & 0,53 & 7,52 & 4,18 \\
MOLLUSCA & & & & \\
Pteropoda & 0,53 & 0,02 & 2,21 & 0,80 \\
PISCES & & & & \\
E. ringens & 0,03 & 1,87 & 0,44 & 0,61 \\
Vinciguerria sp. & 5,09 & 46,63 & 27,88 & 7,27 \\
Myctophidae & 0,03 & 0,71 & 0,44 & 0,28 \\
Teleósteos indeterminados & 1,60 & 16,52 & 4,87 & 4,49 \\
\hline
\end{tabular}

Tabla 3. Porcentaje en número $(\% \mathrm{~N})$, porcentaje en peso $(\% \mathrm{P})$, porcentaje de frecuencia de ocurrencia (\%F) e índice de importancia relativa por ítemes presa de jurel en otoño de 1994.

Table 3. Percentage in numbers $(\% \mathrm{~N})$, in weight $(\% \mathrm{P})$, of occurrence frequency $(\% \mathrm{~F})$ and index of relative importance, by prey taxon of jack mackerel for the autumn 1994.

\begin{tabular}{|lrrrr|}
\hline PRESAS & \% N & $\mathbf{\% P}$ & $\mathbf{\% F}$ & IIR \\
\hline CRUSTACEA & & & & \\
Copepoda & 9,81 & 0,19 & 1,00 & 2,40 \\
Ostracoda & 0,02 & 0,00 & 0,25 & 0,01 \\
Amphipoda & 30,78 & 0,95 & 6,02 & 5,26 \\
Euphausiacea & 42,32 & 15,99 & 56,64 & 8,10 \\
Emerita sp. & 3,30 & 0,21 & 2,26 & 2,19 \\
Blepharipoda & 0,62 & 0,02 & 1,50 & 0,67 \\
Galatheidae & 4,69 & 1,44 & 5,51 & 3,55 \\
Porcellanidae & 0,10 & 0,00 & 1,00 & 0,10 \\
Brachyura & 7,24 & 0,53 & 7,52 & 4,08 \\
MOLLUSCA & & & & \\
Cephalopoda Teuthoidea & 0,01 & 0,52 & 0,25 & 0,12 \\
PISCES & & & & \\
E. ringens & 0,98 & 74,84 & 14,79 & 7,02 \\
Vinciguerria sp. & 0,02 & 0,34 & 0,50 & 0,17 \\
Scomberexos sp. & 0,02 & 0,27 & 0,50 & 0,14 \\
Teleósteos indetermin. & 0,10 & 4,69 & 2,26 & 2,47 \\
\hline
\end{tabular}


Tabla 4. Porcentaje en número $(\% \mathrm{~N})$, porcentaje en peso $(\% \mathrm{P})$, porcentaje de frecuencia de ocurrencia (\%F) e índice de importancia relativa por ítemes presa de jurel en invierno de 1994.

Table 4. Porcentage in numbers $(\% \mathrm{~N})$, in weight $(\% \mathrm{P})$, of occurrence frequency $(\% \mathrm{~F})$ and index of relative importance, by prey taxon of jack mackerel for the winter 1994.

\begin{tabular}{|lrrrr|}
\hline PRESAS & \%N & $\mathbf{\% P}$ & $\mathbf{\%}$ & IIR \\
\hline CRUSTACEA & & & & \\
Estomatopoda & 0,01 & 0,03 & 0,52 & 0,02 \\
Euphausiacea & 97,42 & 42,63 & 65,10 & 9,12 \\
Penaeidae & 0,13 & 1,51 & 1,04 & 1,00 \\
Emerita sp. & 0,34 & 0,03 & 2,08 & 0,58 \\
Anomura & 0,02 & 0,00 & 0,52 & 0,01 \\
Brachyura & 0,23 & 0,02 & 1,56 & 0,33 \\
MOLLUSCA & & & & \\
Cephalopoda Teuthoidea & 0,01 & 0,34 & 0,52 & 0,17 \\
PISCES & & & & \\
E. ringens & 0,15 & 33,72 & 6,77 & 5,44 \\
Vinciguerria sp. & 1,15 & 7,17 & 4,17 & 3,57 \\
Diogenichthys sp. & 0,20 & 0,67 & 1,56 & 0,86 \\
Teleósteos indetermin. & 0,32 & 13,88 & 16,15 & 5,44 \\
\hline
\end{tabular}

nores a $21 \mathrm{~cm}$ de LH se debería al aporte de Vinciguerria sp., Brachyura (zoea y megalopa) y Copepoda. Las siguientes unidades ontogenéticas tróficas se caracterizaron por la presencia de los mismos taxa de presas (Euphausiacea, E. ringens y Vinciguerria sp.), aunque se segregaron por los diferentes aportes relativos de cada uno de ellos.

En verano de 1994, el jurel presentó dos unidades tróficas ontogenéticas, segregadas alrededor de los $25 \mathrm{~cm}$ de LH. La diferencia se debió al menor aporte de eufáusidos en los ejemplares menores a 26 cm de LH, con relación al grupo formado por los ejemplares más grandes. Sin embargo, es posible que esta diferencia cuantitativa en la constitución de asociaciones tróficas se deba al escaso número de observaciones, i.e. bajo número de estómagos con contenido en ejemplares menores de $26 \mathrm{~cm}$ de LH. La similitud trófica promedio entre los grupos de tallas del jurel en verano de 1994 (IS = 0,73) fue bastante mayor que en primavera (IS $=0,56$ ) (Fig. 2b).

En otoño de 1994, el jurel presentó dos unidades ontogenéticas tróficas (Fig. 2c), una constituida
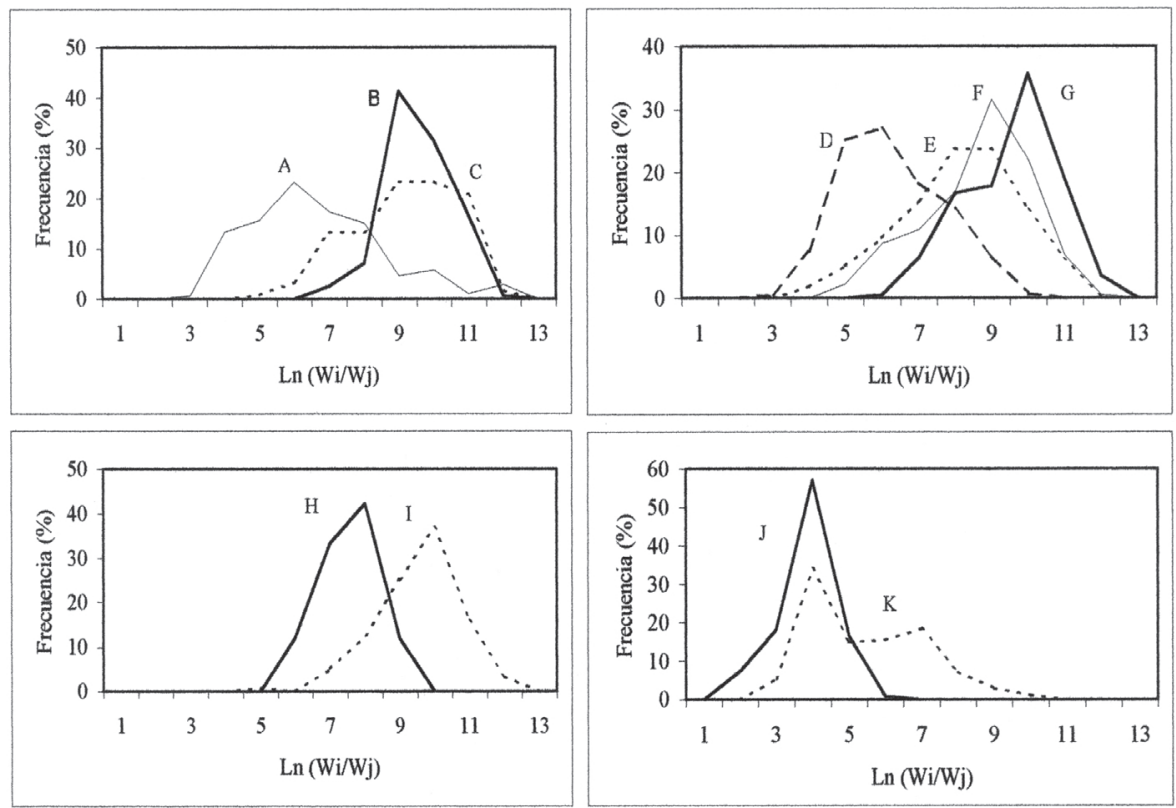

Figura 3. Distribución de frecuencias del índice de selectividad de Ursin de las principales presas de jurel en el norte de Chile. A: Copepoda, B: Ostracoda, C: Amphipoda, D: Euphausiacea, E: Emerita, F: Brachyura (megalopa), G: Brachyura (zoea), H: Chaetognatha, I: Pteropoda, J: Engraulis ringens, y K: Vinciguerria sp.

Figure 3. Frequency distributions of Ursin's index for the most important preys of jack mackerel in northern Chile. A: Copepoda, B: Ostracoda, C: Amphipoda, D: Euphausiacea, E: Emerita, F: Brachyura (megalopa), G: Brachyura (zoea), H: Chaetognatha, I: Pteropoda, J: Engraulis ringens, and K: Vinciguerria sp. 
por individuos menores a $21 \mathrm{~cm}$ de LH y otra por individuos entre 21 y $35 \mathrm{~cm}$. Esta segregación se debería al aporte de E. ringens en individuos mayores a $20 \mathrm{~cm}$ de LH. La similitud trófica promedio en esta estación fue de 0,56 , similar a la de verano. Por último, en invierno de 1994, el jurel presentó dos unidades ontogenéticas tróficas, segregadas alrededor de los $30 \mathrm{~cm}$ de LH (Fig. 2d). La diferencia se debió al mayor aporte de E. ringens en ejemplares mayores a $30 \mathrm{~cm}$ de LH. La similitud trófica media fue de 0,51 .

Las distribuciones de frecuencias de los valores del índice de selectividad de Ursin (ISU) de los taxa presas con mayor número de valores ISU calculados se graficaron independientemente por taxon (Fig. 3). Los eufáusidos y Vinciguerria sp. se eliminaron del análisis de selectividad por no presentar una distribución normal (Test KolmorogovSmirnov). La comparación entre las medias de los valores ISU mediante el análisis de varianza, muestra que éstas son significativamente diferentes $(\mathrm{p}<$ $0,00001)$ y la comparación múltiple indica que $E$. ringens es la presa de mayor tamaño, en relación al peso del predador, siendo significativamente diferente al resto $(\mathrm{p}<0,05)$. Los taxa presa Amphipoda, Ostracoda, zoea de Brachyura y Pteropoda, conforman un grupo homogéneo de presas de tamaño similar, no encontrándose diferencias significativas entre sus medias (Tabla 5). Las desviaciones estándar

Tabla 5. Comparaciones de los ISU promedios de las principales presas de jurel en la zona norte de Chile. (Test-Duncan, $\alpha=0,05$ ), $\mathrm{N}$ : número de observaciones, línea oscura: grupos homogéneos sin diferencias significativas entre promedios.

Table 5. Comparison of the average ISU for the main preys of jack mackerel (Duncan's Test, $\alpha=0.05$ ). N: number of observations, dark line: homogeneous groups, there are no significant differences.

\begin{tabular}{|lrcc|}
\hline Presas & N & $\begin{array}{c}\text { ISU } \\
\text { Pro- } \\
\text { medio }\end{array}$ & $\begin{array}{c}\text { Grupos } \\
\text { Homo- } \\
\text { géneos }\end{array}$ \\
\hline Engraulis ringens & 121 & 3,328 & \\
Copepoda & 172 & 6,150 & \\
Chaetognatha & 33 & 7,016 & \\
Emerita sp. & 156 & 7,632 & \\
Brachyura (megalopa) & 218 & 8,127 & \\
Amphipoda & 120 & 8,670 & \\
Ostracoda & 155 & 9,019 & \\
Brachyura (zoea) & 169 & 9,033 & \\
Pteropoda & 119 & 9,053 & \\
\hline
\end{tabular}

del ISU de E. ringens y Chaetognatha no son significativamente diferentes y a la vez presentan las menores desviaciones estándar, lo que se interpreta como que el jurel selecciona por tamaño principalmente a E. ringens y Chaetognatha (bulk prey). Además, Copepoda fue el taxon menos seleccionado y tuvo la mayor desviación estándar $(\mathrm{DE}=1,946)$ (Tabla 6).

\section{DISCUSIÓN}

En el norte de Chile, T. s. murphyi presentó un amplio espectro de presas sólo en primavera, reduciéndose éste fuertemente en las otras estaciones del año. Sin embargo, los pocos taxa de presas importantes son consistentemente los mismos en las cuatro estaciones (Euphausiacea, Vinciguerria sp., larvas de crustáceos y E. ringens), coincidiendo parcialmente con lo informado por Medina (1990) y Medina y Arancibia (1992). Sin embargo, Galaz (1992) registró para la misma zona a los eufáusidos como la presa principal, con Vinciguerria sp. como especie incidental. Por otro lado, Cornejo-Rodríguez (1991) y Arancibia et al. (1995), en estudios de alimentación del jurel del centro-sur de Chile en 1990 y 1994 respectivamente, encontraron que también los eufáusidos fueron la presa principal, siendo secundarios los "peces linterna". Recientemente, Miran-

Tabla 6. Comparación de las desviaciones estándar (DE) de los ISU de las principales presas de jurel en la zona norte de Chile. (Test-F, $\alpha=0,05$ ), $\mathrm{N}$ : número de observaciones, línea oscura: grupos homogéneos sin diferencias significativas entre las $\mathrm{DE}$.

Table 6. Comparison of the standard deviations (DE) of the ISU for the main preys of jack mackerel (FTest, $\alpha=0.05)$. N: number of observations, dark line: homogeneous groups, there are no significant differences.

\begin{tabular}{|c|c|c|c|}
\hline Presas & $\mathbf{N}$ & DE & $\begin{array}{c}\text { Grupos } \\
\text { homogéneos }\end{array}$ \\
\hline Engraulis ringens & 121 & 0,730 & \\
\hline Chaetognatha & 33 & 0,866 & \\
\hline Ostracoda & 155 & 0,932 & \\
\hline Pteropoda & 119 & 1,242 & \\
\hline Brachyura (zoea) & 169 & 1,276 & \\
\hline Brachyura (megalopa) & 218 & 1,415 & \\
\hline Amphipoda & 120 & 1,475 & | \\
\hline Emerita sp. & 156 & 1,595 & 1 \\
\hline Copepoda & 172 & 1,946 & \\
\hline
\end{tabular}


da et al. (1998) determinaron que el jurel de la zona centro-sur presenta no más de dos especies de presas dominando en la alimentación, siendo los eufáusidos el principal ítem alimentario durante el invierno de 1994.

En el jurel se revela la constitución de más de una unidad ontogenética trófica, con el núcleo entre 21 y $35 \mathrm{~cm}$ de longitud horquilla (rango de tamaño analizado: 10 a $50 \mathrm{~cm} \mathrm{LH}$ ). Algunos cambios en estos límites podrían deberse al efecto de muestras pequeñas de estómagos y/o a la falta de simultaneidad de éstas en el tiempo y espacio como a la disponibilidad de presas presente en las estaciones del año analizadas. La segregación trófica a los $21 \mathrm{~cm}$ LH podría estar relacionada con procesos fisiológicos de madurez sexual, la que fue calculada en 21,6 $\mathrm{cm}$ de longitud horquilla para el jurel de la zona norte de Chile (Alegría et al., 1995), y en 21,3 cm para ejemplares del Perú (Dioses et al., 1989). Medina (1990) y Medina y Arancibia (1992) no encontraron ninguna segregación trófica con el crecimiento del jurel en el norte de Chile (rango de tamaño analizado: 25 a $36 \mathrm{~cm} \mathrm{LH}$ ), y algo similar encontró Arancibia (1989) para esta misma especie en la zona centro-sur (rango de tamaño analizado: $23 \mathrm{a} 44 \mathrm{~cm})$.

Por otro parte, Miranda et al. (1998) encontraron diferencias en la alimentación por tamaño, en un rango de 26 a $55 \mathrm{~cm}$ de $\mathrm{LH}$, y también por zona de pesca, diferencias que estuvieron dadas por la presencia de salpas en los grupos de tallas mayores a $31 \mathrm{~cm}$ de LH. Estas discrepancias podrían estar asociadas con las estrategias de muestreo en tiempo y espacio, al tamaño de muestras por rango de talla y/o a las zonas de pesca. Al respecto, Miranda et al. (1998) señalan que la composición alimentaria del jurel y la proporción de organismos en su dieta está determinada por la composición específica en las áreas de alimentación, lo cual es lógico si se considera que el jurel se encaja dentro de la categoría de predador terciario y posee un comportamiento altamente migratorio.

El jurel parece seleccionar por tamaño algunas de sus presas en la zona norte, lo que es especialmente válido para la anchoveta, quetognatos $\mathrm{y}$ ostrácodos, ya que son los taxa que presentan las más bajas desviaciones estándar del índice de selectividad de Ursin y, a la vez, la anchoveta fue la presa de mayor tamaño seleccionada, diferiendo significativamente del resto de los taxa presa. Llama la atención la selección de quetognatos y ostrácodos, por ser especies muy pequeñas y probablemente con bajo aporte energético, aunque ya habían sido señaladas como presas (Konchina, 1980), pero el índice de selectividad por tamaño de Ursin considera el peso de cada taxon en el estómago y no el peso individual de cada presa (Clark, 1985). Además, la selección sobre estos taxa pudo deberse a que en ese período estas especies eran dominantes en el zooplancton, ingiriendo por lo tanto una mayor cantidad de estos zooplancteres. Lamentablemente, no se cuenta con registros de la composición y dominancia zooplanctónica en el área de estudio durante los períodos de muestreos para confirmar esto.

Miranda et al. (1998) son los primeros en comunicar la presencia de salpas en la alimentación del jurel en la zona centro sur, siendo caracterizadas como zooplancteres de escaso valor nutritivo (Fagetti y Fisher, 1964 fide Miranda et al., 1998), e indican que la ingesta de salpas puede ser considerada incidental debido a su asociación con Euphausia mucronata en sectores costeros y a la alta densidad observada durante el período de estudio. Los copépodos fueron la presa menos seleccionada en todo el período. Los eufáusidos y Vinciguerria sp. no mostraron una distribución normal en el índice de selectividad, lo cual se explicaría con el postulado de Ursin (1973), que señala que la distribución de frecuencias del índice se aparta de una distribución normal cuando los recursos presa son abundantes en la naturaleza. Aunque en este estudio no se han identificado los eufaúsidos, Antezana (1970) afirma que Euphausia mucronata es la especie más frecuente y abundante en sectores costeros. Quiñones et al. (1997) analizaron la relación espacial entre el jurel y su principal presa, los eufáusidos, en la zona centro-sur de Chile, encontrando que esta especie presentó la mayor abundancia en la zona nerítica. Ellos concluyen que a nivel de mesoescala, la distribución espacial entre el jurel y los eufáusidos permite que el recurso encuentre alimento sin mayor dificultad en la zona de estudio.

Aunque la anchoveta no es una presa frecuente en la alimentación del jurel en la zona norte, es interesante comparar el tamaño de los individuos presa con los capturados por la flota industrial de cerco en el mismo período. Al respecto, el tamaño de las anchovetas presas del jurel varió entre 9 y $16 \mathrm{~cm}$ de LT (Fig. 4), encontrándose ausentes en los estómagos de jurel los tamaños de anchoveta que se reclutan a la pesquería, que corresponden a individuos de $7,2 \mathrm{~cm}$ de LT (Cubillos, 1991), lo que probablemente se debe a una segregación espacial entre el 

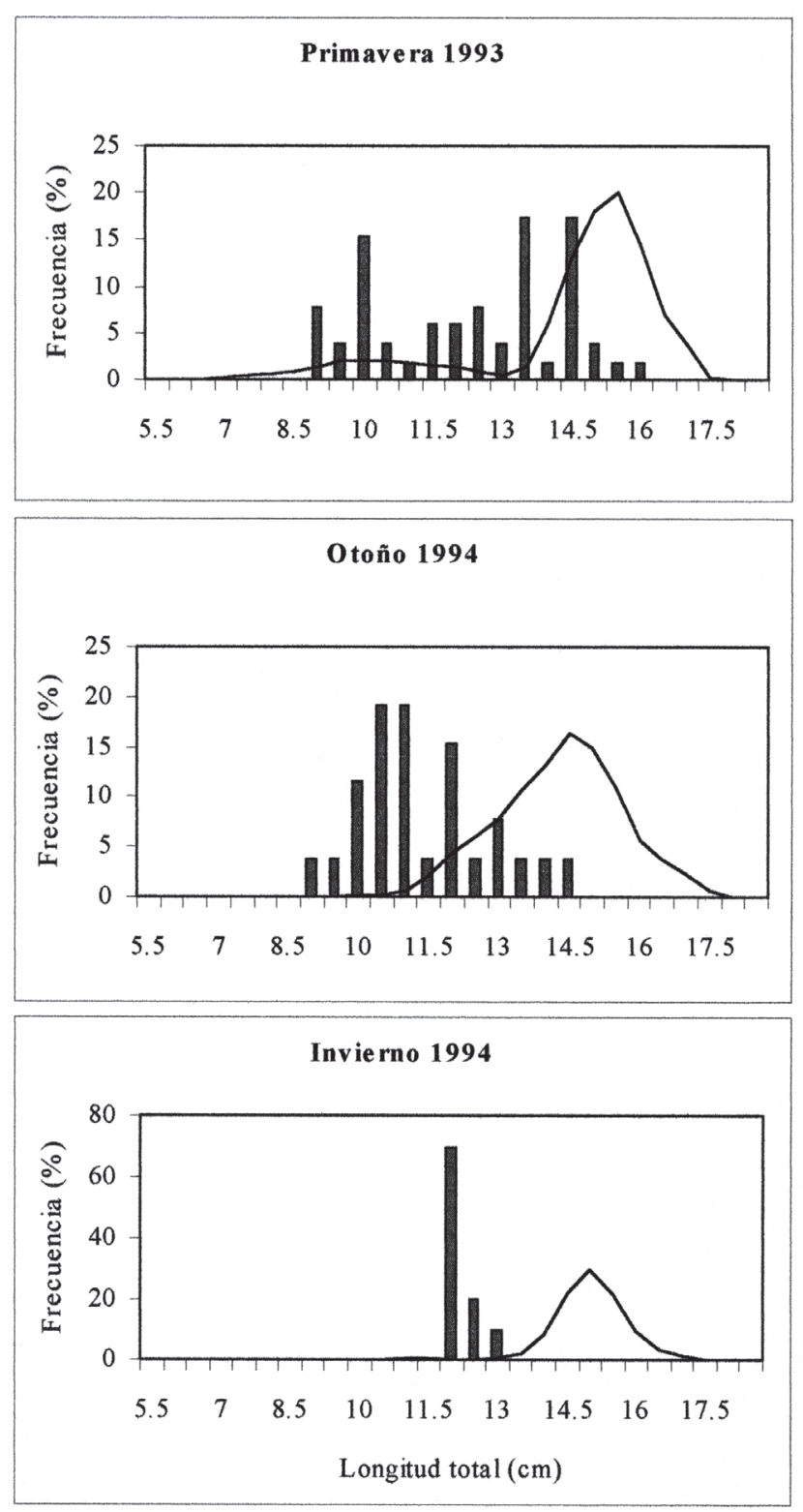

Figura 4. Comparación estacional de las distribuciones de frecuencia de tallas de Engraulis ringens en los estómagos de jurel (barras) y de la pesquería (línea continua) en el norte de Chile.

Figure 4. Seasonal comparison of the size frequency distributions of Engraulis ringens in the stomachs of jack mackerel (bars) and from the fishery (continuos line) in northern Chile. jurel y la anchoveta. Además, los ejemplares de anchoveta mayormente predados por el jurel no coinciden con la moda que es principalmente afectada por la pesquería $(14,5$ a $15,5 \mathrm{~cm})$. La predación sobre anchoveta ocurriría en áreas costeras, como lo indican las zonas de pesca (10 a $40 \mathrm{mn}$ de la costa) de donde se obtuvieron las muestras de estómagos de jurel para este estudio. Contrariamente, Vinciguerria sp. y otros peces mesopelágicos fueron predados en el sector oceánico, ya que la mayor distribución de este tipo de presas en la zona norte de Chile ocurre a alrededor de las $60 \mathrm{mn}$ de la costa hacia el oeste (Palma, 1993). Esto confirma que el jurel presenta un carácter de predador facultativo heterotrófico con habilidad de habitar diferentes biotopos (sensu Konchina, 1980), de tal manera que la composición alimentaria va a estar determinada por el espectro de especies presentes en las áreas de alimentación.

\section{AGRADECIMIENTOS}

Se agradece al Fondo de Investigación Pesquera por el financiamiento del Proyecto FIP 93-18 "Estudio biológico pesquero del recurso jurel en la zona norte de Chile (Regiones I y II)"; al Sr. Mauricio Vargas y a la Sra. María Inés Tapia por el análisis de las muestras, y al Sr. Miguel Araya por su aporte crítico al manuscrito.

\section{REFERENCIAS}

Alegría, V., J. Oliva, H. Robotham, H. Miranda, G. Böhm, L. Caballero, D. Boré, U. Parker, V. Baros, S. Peña, G. Claramunt, G. Herrera, C. Padilla, P. Pizarro, M. Medina, H. Arancibia, M. Araya, L. Cubillos, R. Gili, D. Garland, H. Hidalgo y M. Oliva. 1995. Proyecto FIP 93-18 "Estudio biológico pesquero sobre el recurso jurel en la zona norte de Chile (Regiones I y II)". Informe Final. Fondo de Investigación Pesquera. Instituto de Fomento Pesquero, 221 pp. 
Antezana, T. 1970. Eufáusidos de la costa de Chile. Su rol en la economía del mar. Rev. Biol. Mar., Valparaíso, 14(2): 19-27.

Arancibia, H. 1989. Distribution patterns, trophic relationships and stock interactions in the demersal fish assemblage off central Chile. Dr. rer. nat. Tesis. University of Bremen, RFA, 221 pp.

Arancibia, H. 1991. Análisis ecológico-pesquero del recurso langostino colorado (Pleuroncodes monodon) y su interacción con merluza común (Merluccius gayi) y lenguado de ojos grandes (Hippoglossina macrops). Biol. Pesq., 20: 37-48.

Arancibia, H., L. Miranda, S. Núñez, G. Vásquez y E. Yáñez. 1995. Determinación de las relaciones tróficas del recurso jurel. En: H. Arancibia, L. Vilugrón y D. Arcos (eds.). Estudio biológico pesquero sobre el recurso jurel en la zona centrosur, V a IX Regiones. Informe Final, Proyecto FIP 94-19. Instituto de Investigación Pesquera Octava Región, Chile, 260 pp.

Bax, N. J. 1998. The significance and prediction of predation in marine fisheries. ICES J. Mar. Sci., 55: 997-1030.

Bloom, S.A. 1981. Similarity indices in community studies: potential pitfalls. Mar. Ecol. Prog. Ser., 5: 125-128.

Caddy, J.F. y G.D. Sharp. 1988. Un marco ecológico para la investigación pesquera. FAO Doc. Téc. Pesca, 283: 1-155.

Clark, M.R. 1985. The food and feeding of seven fish species from the Campbell Plateau, New Zeland. J. Mar. Freshw. Res., 19: 339-363.

Cornejo-Rodríguez, M.H. 1991. Causas posibles de las agregaciones de jurel (Trachurus murphyi, Nichols): disponibilidad de alimento. Tesis de Magister en Ciencias con Mención en Oceanografía. Universidad de Concepción, Concepción, 108 pp.

Cubillos, L. 1991. Estimación mensual, reclutamiento y mortalidad por pesca de la anchoveta (Engraulis ringens) de la zona norte de Chile en el período 1986-1989. Biol. Pesq., 20: 49-59.

Daan, N. y M.P. Sissenwine (eds). 1991. Multispecies models relevant to management of living resources (ICES Marine Science Symposia). ICES J. Mar. Sci., 193: 1-358.
Dioses, T., V. Alarcón, M.H. Nakama y A. Echeverría. 1989. Desarrollo ovocitario, fecundidad parcial y distribución vertical de los cardúmenes en desove del jurel Trachurus murphyi (N). En: R. Jordán, R. Kelly, O. Mora, A. de Vildoso y N. Henríquez (eds.). Memorias del Simposio internacional sobre recursos vivos y pesquerías en el Pacífico sudeste. Rev. Com. Perm. Pacífico Sur, Número especial, pp. 287-294.

Galaz, L. 1992. Análisis trófico comparado del jurel (Trachurus symmetricus murphyi Nichols, 1920) de las zonas de pesca del norte y centro-sur de Chile. Tesis de Biólogo Pesquero. Universidad Arturo Prat, Iquique, 82 pp.

Gislason, H. y P. Sparre. 1987. Some theoretical aspects of the implementation of Multispecies Virtual Population Analysis in ICES. ICES C.M./G:51 Demersal Fish Cttee., 39 pp.

Grechina, A. 1992. Historia de investigación y aspectos básicos de la ecología del jurel (Trachurus symmetricus murphyi) en alta mar del Pacífico Sur. En: H. Arancibia y L. Cubillos (eds.). Documento Técnico. Instituto de Investigación Pesquera, Talcahuano, 1(2): 1-47.

Hyslop, E.J. 1980. Stomach contents analysis. A review of methods and their applications. J. Fish. Biol., 17: 411-429.

Konchina, G.V. 1980. The Peruvian jack mackerel, Trachurus symmetricus murphyi, a facultative predator in the coastal upwelling ecosystem. J. Ichthyol., 20(5): 46-59.

Konchina, G.V. 1983. The feeding niche of the hake Merluccius gayi (Merlucciidae), and the jack mackerel, Trachurus symmetricus (Carangidae) in the trophic system of the Peruvian coastal upwelling. J. Ichthyol., 23(2): 87-98.

Livingston, R.J. 1982. Trophic organization of fishes in a coastal seagrass system. Mar. Ecol. Prog. Ser., 7: 1-12.

Medina, M. 1990. Interacciones tróficas entre el jurel (Trachurus murphyi, Nichols) y la caballa (Scomber japonicus, Jordán y Hubbs) en el ecosistema pelágico de la zona norte de Chile. Tesis de Biólogo Pesquero. Universidad Arturo Prat, Iquique, $76 \mathrm{pp}$.

Medina, M. y H. Arancibia. 1992. Interacciones tróficas entre el jurel (Trachurus murphyi) y la caballa (Scomber japonicus) en el ecosistema pelá- 
gico de la zona norte de Chile. Invest. Cient. Tecnol., Ser. Cienc. Mar, 2: 67-78.

Miranda, L., A. Hernández, A. Sepúlveda y M. Landaeta. 1998. Alimentación de jurel y análisis de la selectividad en la zona centro-sur de Chile. En: D. Arcos (ed.). Biología y ecología del jurel en aguas chilenas. Instituto de Investigación Pesquera, Talcahuano, $216 \mathrm{pp}$.

Palma, W. (ed.). 1993. Conclusiones científicas del Proyecto "Aplicaciones de nuevas tecnologías para la captura de recursos hidrobiológicos marinos no tradicionales de la Primera Región". Programa Nacional de Cooperación Técnica con el Gobierno de Chile. Universidad Arturo Prat, Iquique, 120 pp.

Pennington, M., R. Bowman y R. Langton. 1982. Variability of the weight of stomach contents of fish and its implications for food studies. En: M. Cacilliet y A. Simenstad (eds.). Gutshop 81. Fish food habits studies. Proceedings of the Third Pacific Workshop Washington Sea Grant Publication. University of Washington, pp. 2-7.

Prenski, L.B. y V. Angelescu. 1993. Ecología trófica de la merluza común (Merluccius hubbsi) del mar argentino. Parte 3. Consumo anual de alimento a nivel poblacional y su relación con la explotación de las pesquerías multiespecíficas. INIDEP Documento Científico 1, Mar del Plata, 118 pp.
Quiñones, R., R. Serra, S. Núñez, H. Arancibia, J. Córdova y F. Bustos. 1997. Relación espacial entre el jurel (Trachurus symmetricus murphyi) y sus presas en la zona centro-sur de Chile. En: E. Tarifeño (ed.). Gestión de Sistemas Oceanográficos del Pacífico Oriental. Comisión Oceanográfica Intergubernamenteal de la UNESCO. IOC/INF1046, 432 pp.

Ross, S.T. 1978. Trophic ontogeny of the leopard sea robin, Prionotus scitulus (Pisces: Triglidae). Fish. Bull., 78: 225-234.

Serra, R. 1991. Important life history aspects of the chilean jack mackerel, Trachurus symmetricus murphyi. Invest. Pesq. (Chile), 36: 67-83.

Schmitter-Soto, J.J. y J.J. Castro-Aguirre. 1996. Trophic comparison among Triglidae (Pisces: Scorpaeniformes) off Baja California Sur, México. Rev. Biol. Trop., 44: 803-811.

Sneath, P.H.A. y R. Sokal. 1973. Numerical taxonomy. W.H. Freeman and Co., New York, 573 pp.

Ursin, E. 1973. On the prey size preference of cod and dab. Meddr. Danm. Fisk.-og Havunders., 7: 85-98.

Walters, C., V. Christensen y D. Pauly. 1997. Structuring dynamic models of exploited ecosystems from trophic mass-balance assessments. Rev. Fish Biol. Fish., 7: 139-172. 